\section{Mit Workfare aus der Sozialhilfe? Lehren aus einem Modellprojekt}

\author{
Hilmar Schneider • Arne Uhlendorff • \\ Klaus F. Zimmermann
}

Angenommen: 4. Februar 2011 / Online publiziert: 22. März 2011

(C) Institut für Arbeitsmarkt- und Berufsforschung 2011

Zusammenfassung Wir berichten über die Evaluation des Pilotprojektes eines Berliner Sozialamts zur Integration junger Sozialhilfeempfänger in das Erwerbsleben. Dem Workfare-Prinzip folgend war die Teilnahme an dem Programm für die ausgewählten Personen verpflichtend. Wer die Teilnahme verweigerte, erhielt keine Unterstützungszahlungen. Um die Wirkung des Programms untersuchen zu können, sollten Engpässe bei der Programmkapazität als quasi-zufälliger Zuweisungsprozess genutzt werden. Im Nachhinein stellte sich jedoch heraus, dass im Zuweisungsprozess unkontrollierte Selektionseffekte auftraten, die den Vergleich mit einer entsprechenden Gruppe von Nichtteilnehmern erheblich erschweren. Mit Hilfe ökonometrischer Verfahren haben wir versucht, für diese Selektion zu korrigieren. Die Messung der Wirkung beruht auf Sozialamtsdaten, die von uns mit Geschäftsdaten der Bundesagentur für Arbeit verknüpft wurden, um Informationen über Erwerbsverläufe vor und nach der Betreuung durch das Sozialamt zu gewinnen. Die Ergebnisse deuten auf einen positiven statistisch allerdings nicht signifikanten - Beschäftigungseffekt von Workfare hin. Wir verdeutlichen, dass Nachlässigkeiten im Projektdesign durch ökonometrische Verfahren zwar nachträglich ausgeglichen werden können, aber nur um

\footnotetext{
A. Uhlendorff

Universität Mannheim, Mannheim, Deutschland

H. Schneider $(\varangle) \cdot$ A. Uhlendorff · K.F. Zimmermann IZA, Schaumburg-Lippe-Str. 5-9, 53113 Bonn, Deutschland e-mail: schneider@iza.org
}

H. Schneider · A. Uhlendorff - K.F. Zimmermann DIW Berlin, Mohrenstraße 58, 10117 Berlin, Deutschland

K.F. Zimmermann · K.F. Zimmermann Universität Bonn, Bonn, Deutschland den Preis eines deutlich erhöhten Fallzahlbedarfs, um statistisch belastbare Ergebnisse für politische Entscheidungen zu bekommen. Bei den üblicherweise niedrigen Fallzahlen im Rahmen von Modellprojekten sollten daher verstärkt experimentelle Designs Anwendung finden.

Schlüsselwörter Workfare · Arbeitsangebot - Evaluation · Soziale Experimente

JEL Klassifikationen J22 $\cdot$ J64 $\cdot$ H43

\section{Does welfare to work work? Lessons from a pilot project}

Abstract We are reporting from an evaluation of a municipal workfare pilot project. The program was intended to integrate young welfare recipients into employment. According to the workfare principle, participation was mandatory for those being assigned to the program. Welfare claims were denied for those who refused to participate. Since program capacity was limited, the assignment process could be viewed as a quasi-random process, which allowed for an impact evaluation by comparing program participants with recipients not assigned to the program due to capacity limitations. In retrospect, however, it turned out that the assignment process was still subject to uncontrolled selection, which strongly impedes comparability between treated and alleged controls. By means of econometric methods we have tried to correct for selectivity. Impact measurement is based on administrative data of the welfare authority which were merged with administrative data from the Federal Labor Agency in order to get information about employment histories before and after welfare claiming. The results are pointing to a strong positive employment effect of workfare, 
although not statistically significant. We conclude that slackness of project design may be compensated by econometric methods, but only at the price of a strongly increased number of observations in order to achieve statistically significant results. If policy makers need to draw conclusions from typically small scale pilot projects, there is no alternative to a project design, which allows for a controlled experiment.

Keywords Workfare · Labor supply · Evaluation · Social experiment

\section{JEL Classification J22 $\cdot$ J64 $\cdot$ H43}

\section{Einleitung}

Das deutsche System der sozialen Mindestsicherung in Form von Arbeitslosen- und Sozialhilfe generiert implizite Mindestlöhne und somit negative Anreizwirkungen auf das Arbeitsangebot im unteren Einkommensbereich. Bei der sozialen Mindestsicherung in Form von Arbeitslosengeld II und Sozialhilfe handelt es sich um einen Zahlungsanspruch, der bei Erfüllung der Bedürftigkeitskriterien zeitlich praktisch unbegrenzt ist. Die Höhe des Transferbezugs sowie die nahezu vollständige Anrechnung des eigenen Erwerbseinkommens auf den Transferbezug determinieren einen impliziten Mindestlohn. Übersteigt dieser Mindestlohn den am Markt erzielbaren Lohn, was vor allem bei gering qualifizierten Erwerbspersonen der Fall sein dürfte, ist die Inanspruchnahme von Transferzahlungen attraktiver als die Ausübung einer Erwerbstätigkeit, selbst wenn das erzielbare Erwerbseinkommen über der Höhe des Transferanspruchs liegt und damit eine eigenständige Existenzsicherung möglich wäre. Je höher die Transferzahlungen und damit der Mindestlohn, desto größer ist der Anreiz, auf eine Erwerbstätigkeit zu verzichten und stattdessen Transferzahlungen in Anspruch zu nehmen (siehe beispielsweise Christofides 2000 oder Schneider und Uhlendorff 2005). Trotz dieser negativen Anreize kann man im deutschen Transfersystem sogenannte „Aufstocker“ beobachten. Die weitaus meisten von ihnen arbeiten jedoch nur in relativ geringem zeitlichen Umfang und verhalten sich somit durchaus anreizkonform, da die so erzielten Einkommen weitgehend anrechnungsfrei bleiben. Der Anteil der Personen, die einer Vollzeitbeschäftigung nachgehen bei der sie weniger als die Grundsicherung verdienen und die daher zusätzliche Transferleistungen beziehen, ist dagegen sehr gering (Brenke und Ziemendorff 2008).

Eine mögliche Option, mit der sich einerseits die hohen Belastungen für die öffentlichen Haushalte reduzieren lassen und die andererseits den Betroffenen Perspektiven für ein unabhängiges Einkommen vermittelt, besteht in einer Kopplung des Transferanspruchs an die Verpflichtung zur
Ausübung einer Erwerbstätigkeit (Bonin et al. 2007). Dieses auch als Workfare bezeichnete Konzept geht auf amerikanische Vorbilder zurück (vgl. z. B. Ochel 2003) und bezieht seine Wirkung aus einer Verschlechterung des Transfernutzens, ohne die Einkommensposition des Hilfebedürftigen zu beeinträchtigen. Mit Transfernutzen ist hierbei der Nutzen gemeint, den Personen während des Bezugs von Transferleistungen realisieren. Im theoretischen Modell hängt dieser Nutzen von der Höhe des Einkommens und vom Umfang der Freizeit ab. Dies führt dazu, dass niedrig entlohnte Tätigkeiten am Markt akzeptabel werden, denn im Vergleich zur Situation ohne Workfare ist eine Beschäftigung mit einer Entlohnung, die nur geringfügig über dem Transfereinkommen liegt, nun deutlich attraktiver. Eine Alternative zur Reduktion des Anreizproblems stellen vorhandene Sanktionsmöglichkeiten beim Bezug von Arbeitslosengeld II dar, siehe beispielsweise Boockmann et al. (2009) für eine entsprechende empirische Analyse.

In Deutschland ist das Workfare-Prinzip bislang erst vereinzelt eingeführt worden und entsprechende Evaluationsstudien liegen bisher nicht vor. Die Ergebnisse von Mikrosimulationsstudien deuten aber auf erhebliche positive Effekte auf das Arbeitsangebot hin (Bonin und Schneider 2006). Diese positiven Effekte auf das Arbeitsangebot werden von Laborexperimenten bestätigt. Sie zeigen zusätzlich, dass die Akzeptanz von Workfare - zumindest unter den im Experiment geltenden Bedingungen - bei den Teilnehmern am Experiment sehr deutlich ist (Falk und Huffman 2007).

Dieser Beitrag diskutiert das Modellprojekt „Arbeit Sofort!“, bei dem der Bezug von Sozialhilfe an die Aufnahme einer Tätigkeit im Rahmen eines öffentlich geförderten Beschäftigungsprojekts gebunden wurde. ${ }^{1}$ Das Projekt wurde vom Herbst 2002 bis zum Sommer 2003 im Berliner Bezirk Charlottenburg-Wilmersdorf durchgeführt.

Sein Ziel bestand in der Erhöhung der Beschäftigungswahrscheinlichkeit der Teilnehmer. Für die Evaluation von „Arbeit sofort!“ werden daher die Erwerbsverläufe der Teilnehmer mit den Erwerbsverläufen einer geeigneten Gruppe von Sozialhilfeempfängern verglichen, die nicht an „Arbeit sofort!" teilgenommen haben. Beschäftigungsinformationen liegen nur in eingeschränkter Form in den Sozialamtsdaten vor, weshalb die Personendaten aus dem Sozialamt mit Geschäftsdaten der Bundesagentur für Arbeit in einem anonymisierten Verfahren verknüpft wurden.

Wir stellen in Abschn. 2 zunächst das Projekt „Arbeit Sofort!“ vor. Abschn. 3 referiert unsere Datenaufnahme, Abschn. 4 berichtet über die Evaluationsergebnisse. Die Schlussfolgerungen in Abschn. 5 stellen die Befunde in einen wirtschaftspolitischen Zusammenhang und reflektieren, was für die Verbesserung des Erkenntnisfortschritts getan werden muss.

\footnotetext{
${ }^{1}$ Eine ausführliche Darstellung unserer Evaluation von „Arbeit Sofort!“ findet sich in Schneider et al. (2010).
} 


\section{Modellprojekt „Arbeit Sofort!““}

Im Berliner Bezirk Charlottenburg-Wilmersdorf wurde das Projekt „Arbeit Sofort!“ in der Zeit vom 01.09.2002 bis zum 30.08.2003 durchgeführt. Erwerbsfähige im Alter von 18 bis 25 Jahren, die einen Neuantrag auf Sozialhilfe stellten, wurden dem Projekt direkt zugewiesen. Ziel von „Arbeit sofort!" war, durch Integration der Teilnehmer in den ersten Arbeitsmarkt ihre Hilfsbedürftigkeit zu überwinden und den so genannten Sozialhilfekarrieren vorzubeugen. Das Programm bestand aus Qualifizierungs- und Beschäftigungsmodulen und wurde gemeinsam vom Bezirksamt Charlottenburg-Wilmersdorf und dem Arbeitsamt finanziert.

Die Teilnehmer wurden am Tag der Sozialhilfeantragstellung direkt an den Projektträger vermittelt, wo am selben Tag ein Erstgespräch stattfand. Teilnehmer und Projektträger schlossen einen Praktikantenvertrag ab, wobei es sich um ein versicherungspflichtiges Beschäftigungsverhältnis handelte, das auf maximal 6 Monate befristet war. Die Teilnehmer erhielten 434 Euro monatlich zuzüglich eventueller Mietkosten, womit ihre Vergütung geringfügig über dem Sozialhilfeniveau lag. Die Teilnahme an „Arbeit Sofort!“ war nach Zuweisung verpflichtend. Personen, die dennoch nicht an der Maßnahme teilnahmen, erhielten keine Sozialhilfe. Nicht zugewiesen wurden Personen, die eine klare berufliche oder ausbildungsbezogene Zukunftsperspektive hatten und die daher nur vorübergehend Sozialhilfe bezogen.

Die Programmkapazität war auf maximal 100 Personen ausgelegt. Aus dieser Beschränkung ergab sich ein quasizufälliger Zuweisungsmechanismus, da Personen, die zu einem Zeitpunkt einen Antrag auf Sozialhilfe stellten, zu dem die Programmkapazität ausgeschöpft war, nicht an dem Programm teilnehmen konnten. Im Gegensatz zu einem klassischen sozialen Experiment sollten die Antragsteller nicht per Zufallsverfahren in die Teilnehmer- oder die Kontrollgruppe zugewiesen werden. Ist allerdings alleine die Auslastung der Programmkapazität für die Teilnahme entscheidend, dürften sicher unter bestimmten Voraussetzungen die Teilnehmer und Nichtteilnehmer nicht systematisch in ihren Eigenschaften unterscheiden. Die potenzielle $\mathrm{Zu}-$ fälligkeit sollte dazu genutzt werden, durch einen direkten Vergleich von Teilnehmern und Nichtteilnehmern kausale Rückschlüsse auf die Wirksamkeit des Programms ziehen zu können.

Die gesamte Teilnehmerzahl belief sich auf 279 Personen, von denen allerdings nur etwa zwei Drittel in die Analyse eingehen, da zu Beginn des Projekts auch Personen aufgenommen wurden, die bereits seit einiger Zeit Sozialhilfe bezogen hatten. Die Kriterien für die Auswahl von Personen aus dem Bestand der Sozialhilfebezieher waren nicht eindeutig geregelt und dokumentiert. Qualitative Interviews mit den zuständigen Sachbearbeitern haben ergeben, dass diese gezielt auf Personen zugegangen seien, bei denen zu erwarten war, dass sie freiwillig an dem Programm teilnehmen würden. Im Vergleich zu Neueintritten in die Sozialhilfe ist die Bildung einer geeigneten Vergleichsgruppe für diesen Personenkreis daher praktisch nicht möglich.

\section{Datenbasis}

Für die Beurteilung des Maßnahmeneffekts sind Verlaufsdaten erforderlich, da sich der Erfolg zwangsläufig erst im Anschluss an die Maßnahme einstellen kann. Hier besteht das Problem, dass solche Informationen beim Sozialamt nur dann anfallen, wenn Personen im Anschluss an die Maßnahmenteilnahme wieder in die Sozialhilfeabhängigkeit zurück fallen. Eine Befragung von Teilnehmern und Nichtteilnehmern ist auf die Bereitschaft zur Befragungsteilnahme angewiesen, die in der Zielgruppe erfahrungsgemäß sehr gering ist (vgl. beispielsweise Schneider et al. 2002). Daher wurden die erforderlichen individuellen Erwerbsverlaufsinformationen aus den Geschäftsdaten der Bundesagentur für Arbeit (BA) genutzt und mit den Sozialamtsdaten gekoppelt. Hierfür wurden die so genannten Integrierten Erwerbsbiografien (IEB) verwendet (Jacobebbinghaus und Seth 2007). Die IEB enthalten exakte Informationen zu Beschäftigungs- und Arbeitslosigkeitsphasen sowie den entsprechenden Transferleistungen und Löhnen.

Die Gruppe der Teilnehmer umfasst 189, die Gruppe der Nichtteilnehmer 928 Personen. Bei den Nichtteilnehmern handelt es sich um Personen im Alter von 18-25 Jahren, die zwischen September 2002 und August 2003 einen Antrag auf Soziale im Bezirk Charlottenburg-Wilmersdorf gestellt haben, aber nicht an der Maßnahme teilgenommen haben. Die Gruppe der Nichtteilnehmer enthält auch diejenigen Personen, die bereits eine Stelle in Aussicht hatten und deshalb dem Programm nicht zugewiesen wurden. Diese Personen lassen sich nicht identifizieren, da eine entsprechende Information in den Sozialamtsdaten nicht festgehalten wurde. Der einfache Vergleich von Teilnehmern mit Nichtteilnehmern an „Arbeit Sofort!“ ist insofern zu Lasten der Teilnehmer verzerrt. Zudem können Personen, die für die Teilnahme an „Arbeit Sofort!“ ausgewählt wurden, dann aber nicht zum Erstgespräch beim Projektträger erschienen, nicht identifiziert werden, da eine entsprechende Erfassung in den Sozialamtsdaten nicht vorgesehen war. Daher ist es nicht möglich, potentielle Abschreckungseffekte zu ermitteln.

Nicht in jedem Fall ist eine eindeutige Zusammenführung der Information aus den beiden Datenquellen möglich, weshalb für einige der Teilnehmer und Nichtteilnehmer mehr als ein Konto in den Daten der BA zugespielt wurden. Vor Beginn der Datenanalyse wurden daher mehrdeutige Zuordnungen ermittelt und durch Plausibilitätsprü- 
fungen aus dem Datensatz ausgeschlossen. ${ }^{2}$ Aus der IEB wurden anschließend Verlaufsdaten zum Erwerbsstatus der Teilnehmer und Nichtteilnehmer generiert. Die Zielvariable „Ungeförderte Beschäftigung“ ist als Phase sozialversicherungspflichtiger Beschäftigung definiert, während der die Personen keine Leistungen von der Arbeitsagentur bezogen haben, nicht als arbeitslos gemeldet waren und an keiner arbeitsmarktpolitischen Maßnahme teilgenommen haben. Wir betrachten einen Zeitraum von bis zu 12 Monaten nach Eintritt in die Sozialhilfe, was bei einer Programmlaufzeit von bis zu 6 Monaten bedeutet, dass wir die Ergebnisvariable für jeden Teilnehmer somit für mindestens 6 Monate nach Austritt aus dem Programm „Arbeit sofort!“ beobachten.

\section{Befunde}

Qualitative Interviews mit den Sachbearbeitern nach Abschluss des Projekts deuteten darauf hin, dass für den $\mathrm{Zu}-$ gang in die Maßnahme nicht ausschließlich die Auslastung der Programmkapazitäten entscheidend war. Dies bestätigt ein deskriptiver Vergleich verschiedener beobachtbarer Eigenschaften für Teilnehmer und Nichtteilnehmer. So sind die Teilnehmer im Durchschnitt beispielsweise jünger, sie sind seltener verheiratet, haben eine schlechtere Schulausbildung und befanden sich vor Eintritt in die Sozialhilfe zu einem deutlich geringeren Anteil in Beschäftigung. ${ }^{3}$ Die Voraussetzung für aussagefähige Schlussfolgerungen auf der Grundlage eines einfachen Vergleichs von Teilnehmern mit Nichtteilnehmern war somit nicht gegeben.

Vergleicht man die Anteile der Personen zwischen Teilnehmern und Nichtteilnehmern miteinander, die nach 12 Monaten beschäftigt sind, so zeigt sich, dass dieser Anteil unter den Teilnehmern an „Arbeit sofort!“ um ca. 2 Prozentpunkte geringer ausfällt. Da sich beide Gruppen allerdings in beobachteten und möglicherweise auch in unbeobachteten Eigenschaften unterscheiden, kann dieser Unterschied nicht als kausaler Effekt der Maßnahmenteilnahme interpretiert werden. Es ist daher notwendig, die a-prioriUnterschiede zwischen Teilnehmern und Nichtteilnehmern hinsichtlich der Wahrscheinlichkeit für die Aufnahme einer Beschäftigung in geeigneter Form zu berücksichtigen. ${ }^{4}$

In einem ersten Schritt wenden wir Verfahren an, mit denen wir für Unterschiede in beobachtbaren Eigenschaften kontrollieren können. Auf der Grundlage eines einfa-

\footnotetext{
${ }^{2}$ Beispielsweise wurden Datensätze ausgeschlossen, die einen Verdienst aus sozialversicherungspflichtiger Beschäftigung von mehr als $30 €$ am Tag während eines Sozialhilfebezugs auswiesen.

${ }^{3}$ Eine ausführliche Darstellung und Diskussion der Ergebnisse findet sich in Schneider et al. (2010).

${ }^{4}$ Für einen methodischen Überblick über entsprechende Verfahren siehe beispielsweise Lee (2005), Blundell und Dias (2009) oder Imbens und Wooldridge (2008).
}

chen Probit-Modells für die Beschäftigungswahrscheinlichkeit, bei dem selektionsrelevante Merkmale in die Schätzung eingehen, deutet sich im Gegensatz zum einfachen Vergleich der Ergebnisvariablen an, dass die Teilnahme an „Arbeit Sofort!“ die Beschäftigungswahrscheinlichkeit 12 Monate nach Eintritt in die Maßnahme erhöht. Mit 2,1 Prozentpunkten ist der Effekt allerdings nicht sehr groß und statistisch auch nicht signifikant. Alternativ hierzu wenden wir ein Propensity Score Matchingverfahren an, das im Vergleich zum Probit-Modell nicht auf spezifischen parametrischen Annahmen beruht. Beim Matching wird durch die Bildung von statistischen Zwillingen dafür gesorgt, dass der Vergleich der Teilnehmer nur mit Nichtteilnehmern erfolgt, die den Teilnehmern möglichst ähnlich sind. ${ }^{5}$ Der aus dem Matching resultierende Punktschätzer ist wie beim ProbitModell positiv und beträgt 3,5 Prozentpunkte, ist aber ebenfalls nicht signifikant von 0 verschieden.

Beide Methoden beruhen auf der Annahme, dass die Selektion in die Maßnahme aufgrund von beobachteten Merkmalen stattfindet. Die Ergebnisse der qualitativen Interviews mit den Sachbearbeitern deuten jedoch darauf hin, dass für die Maßnahmenzuweisung auch Kriterien eine Rolle spielen, die in den verfügbaren Daten nicht abgebildet sind. Dazu zählen etwa Alkoholabhängigkeit, psychische Probleme, etc. Die Kontrolle einer Selektion auf Grundlage unbeobachteter Eigenschaften ist grundsätzlich möglich, wenn die Selektion in die Maßnahme von einem exogenen Faktor abhängt, der nicht gleichzeitig einen Effekt auf die Zielvariable hat.

In unserem Fall nutzen wir die Tatsache aus, dass die Wahrscheinlichkeit der Maßnahmenteilnahme von der Programmbelegung abhängt, da zu einem Zeitpunkt nicht mehr als 100 Personen an dem Programm teilnehmen konnten. Wir gehen davon aus, dass die Programmbelegung selbst keinen kausalen Einfluss auf die späteren Erwerbschancen hat. Wir schätzen in einem bivariaten Probit Modell die Selektion in „Arbeit sofort!“ gemeinsam mit der Wahrscheinlichkeit, nach 12 Monaten eine Beschäftigung zu haben. Die Programmbelegung wird hierbei in die Schätzung der Teilnahmewahrscheinlichkeit, nicht aber in die Schätzung der Beschäftigungswahrscheinlichkeit aufgenommen. In diesem Modell fällt der marginale Effekt sehr deutlich aus. Teilnehmer an „Arbeit Sofort!“ weisen demnach 12 Monate nach Maßnahmebeginn eine um 27 Prozentpunkte höhere Beschäftigungswahrscheinlichkeit auf als Nichtteilnehmer. Die ökonomische Relevanz der Evaluationsergebnisse spricht für die Wirksamkeit des Workfare-Konzepts. Dennoch ist das Ergebnis aufgrund einer zu geringen Fallzahl aus rein statistischer Sicht nicht ausreichend belastbar. Ein Vergleich der Standardfehler des Maßnahmeneffektes im einfachen Probit Modell mit dem Standardfehler

\footnotetext{
${ }^{5}$ Eine gute Übersicht über die Anwendung von Matching Verfahren geben Caliendo und Kopeinig (2008).
} 
im Modell mit simultaner Selektionskorrektur zeigt, dass der Standardfehler im bivariaten Probit Modell deutlich höher liegt. Die hohe statistische Unsicherheit des Effekts erlaubt daher letztlich keine eindeutigen Schlussfolgerungen über die Wirksamkeit des Workfare-Prinzips in dem vorgestellten Pilotprojekt, denn die Maßnahmenteilnahme hat keinen statistisch signifikanten Effekt auf die Beschäftigungswahrscheinlichkeit. Wie groß eine Stichprobe sein muss, damit eine Parameterschätzung als statistisch signifikant eingestuft werden kann, hängt im Wesentlichen von der Größe des betrachteten Parameters und dessen Stichprobenvarianz ab. Ließe sich die Kovarianz im Rahmen einer MaximumLikelihood Schätzung anhand der Hesse-Matrix bestimmen, müsste der Umfang bei der vorliegenden Parameter Konstellation mindestens zweimal so hoch sein. Da wir robuste Standardfehler berechnen dürfte der Fallzahlbedarf tendenziell sogar noch darüber liegen.

Die gestiegene statistische Unsicherheit verdeutlicht den Preis, der aus einem inkonsequenten Projektdesign resultiert. Die prinzipielle Möglichkeit, mit Hilfe von ökonometrischen Verfahren für Selektionsverzerrungen zu kontrollieren, wird neben der Abhängigkeit von der Gültigkeit der getroffenen Annahmen mit einer erhöhten Parameterstreuung erkauft, was die Aussagefähigkeit der Ergebnisse stark beeinträchtigen kann. Derartige Methoden erfordern in der Regel beträchtlich höhere Fallzahlen als sie im Rahmen von Modellprojekten üblicherweise zur Verfügung stehen.

\section{Schlussfolgerungen}

In unserem Projekt geht es um die Frage, ob die Anwendung des Workfare-Prinzips dazu geeignet ist, die Anreizprobleme der Grundsicherung zu überwinden. Das Ergebnis spricht dafür, dass das Modellprojekt „Arbeit sofort!“ die Beschäftigungswahrscheinlichkeit der Teilnehmer erhöht hat, allerdings werden die üblichen statistischen $\mathrm{Si}$ gnifikanzkriterien auf der Grundlage der verwendeten Daten verfehlt. Dies zeigt, dass insbesondere bei Modellprojekten mit kleinen Fallzahlen die ex-ante-Strategie einer zufälligen Programmzuweisung deutliche Vorteile gegenüber der ex-post-Strategie einer statistischen Selektionskorrektur aufweist.

Im Gegensatz zu aufwändigen ökonometrischen Modellen liefern soziale Experimente selbst bei geringen Fallzahlen einfache und glaubwürdige Schätzungen von Programmeffekten und vermeiden die Abhängigkeit von statistisch nicht überprüfbaren Annahmen (Ashenfelter 1987). Die Durchführung eines Modellprojekts als soziales Experiment erfordert dabei nicht unbedingt eine strikte $\mathrm{Zu}$ weisung im Rahmen eines Zufallsverfahrens. In der Praxis können beispielsweise auch Kapazitätsbeschränkungen als quasi-zufälliger Zuweisungsmechanismus genutzt werden, wie sie im hier vorgestellten Modellprojekt vorlagen. Allerdings dürfen dabei keine Ermessensspielräume zum Tragen kommen. Es müsste sicher gestellt sein, dass alleine die Kapazitätsauslastung für die Teilnahme an der Maßnahme entscheidend ist und keine Selektion der Teilnehmer stattfinden kann.

Gleichwohl sollten soziale Experimente nicht als Königsweg betrachtet werden. Ihren Vorteilen stehen auch Nachteile gegenüber. Dazu gehören relativ hohe Kosten, ethische Bedenken, mögliche Substitutions-, sowie Dropout- und Randomisierungsverzerrungen (siehe bspw. Burtless 1995 oder Heckman und Smith 1995). Höhere Kosten entstehen dabei vor allem durch die Implementierung einer zufälligen Zuordnung von Personen in Teilnehmer- und Kontrollgruppen beispielsweise durch Mitarbeiterschulungen. Soziale Experimente finden oftmals bei kleinen und spezifischen Programmen für bestimmte Zielgruppen Anwendung, die sich in der Ausgestaltung von größeren Programmen unterscheiden. Den Nachteil von mikroökonometrischen Verfahren, die sich im Regelfall auf die Messung des Programmeffekts für die Teilnehmer beschränken und damit Erkenntnisse über allgemeine Gleichgewichtseffekte vernachlässigen, können auch soziale Experimente nur bedingt überwinden. Moffitt (2004) kommt daher zu dem Schluss, dass soziale Experimente mikroökonometrischen Ansätzen nicht generell überlegen seien, sondern sich die Ansätze vielmehr ergänzen können. ${ }^{6}$ Es bleibt allerdings festzuhalten, dass in der deutschen Arbeitsmarktpolitik experimentelle Designs in bisher viel zu geringem Maße Anwendung finden.

Insbesondere bei der Durchführung von Modellprojekten mit üblicherweise kleinen Fallzahlen sollte daher der Aspekt eines erkenntnisorientierten Projektdesigns eine hohe Priorität haben. Die Durchführung von Modellprojekten ohne Chance auf Erkenntnisgewinn stellt eine Vergeudung von Chancen und Ressourcen dar. Für die arbeitsmarktpolitische Politikberatung benötigen wir dringend Erkenntnisse über innovative Modellprojekte, die auf klaren experimentellen Designs beruhen.

Danksagung Wir danken einem anonymen Gutachter und dem Herausgeber für hilfreiche Kommentare. Für ihre finanzielle Unterstützung im Rahmen des Schwerpunktprogramms „Flexibilisierungspotenziale bei heterogenen Arbeitsmärkten“, Teilprojekt ZI 302/7-1 danken wir der Deutschen Forschungsgemeinschaft (DFG). Die Autoren danken ferner den Mitarbeiter des Sozialamtes Berlin-Charlottenburg sowie des Instituts für Arbeitsmarktforschung und Berufsforschung für ihre Unterstützung, Jan Stuhler für seine Mitarbeit und Friedrich-Wilhelm Dopatka, ehemals Staatsekretär im Berliner Senat, für seine Unterstützung bei der Initiierung dieser Evaluationsstudie.

\footnotetext{
${ }^{6}$ Für jüngere und teilweise kontroverse Beiträge zu Nutzen und Grenzen von experimentellen Designs in der empirischen Wirtschaftsforschung siehe beispielsweise Deaton (2009) und Angrist und Pischke (2010).
} 


\section{Literatur}

Angrist, J., Pischke, S.: The credibility revolution in empirical economics: how better research design is taking the con out of econometrics. J. Econ. Perspect. 24(2), 3-30 (2010)

Ashenfelter, O.: The case for evaluating training programs with randomized trials. Econ. Educ. Rev. 6, 333-338 (1987)

Blundell, R., Dias, M.C.: Alternative approaches to evaluation in empirical microeconomics. J. Hum. Resour. 44, 565-640 (2009)

Bonin, H., Schneider, H.: Workfare: eine wirksame alternative zum kombilohn. Wirtschaftsdienst 86, 645-650 (2006)

Bonin, H., Falk, A., Schneider, H.: Workfare - Praktikabel und gerecht. Ifo-Schnelld. 4, 33-37 (2007)

Boockmann, B., Thomsen, S.L., Walter, T.: Intensifying the use of benefit sanctions - an effective tool to shorten welfare receipt and speed up transitions to employment? IZA Discussion Paper 4580 (2009)

Brenke, K., Ziemendorff, J.: Hilfebedürftig trotz Arbeit?: Kein Massenphänomen in Deutschland. Wochenber. - Dtsch. Wirtschaftsforsch. 4, 33-40 (2008)

Burtless, G.: The case for randomized field trials in economic and policy research. J. Econ. Perspect. 9, 63-84 (1995)

Caliendo, M., Kopeinig, S.: Some practical guidance for the implementation of propensity score matching. J. Econ. Surv. 22, 31-72 (2008)

Christofides, L.N.: Social assistance and labour supply. Can. J. Econ. 33, 715-741 (2000)

Deaton, A.: Instruments of development: randomisation in the tropics and the search for the elusive keys to economic development. Proc. Br. Acad. 162, 123-160 (2009)

Falk, A., Huffman, D.: Studying labor market institutions in the lab: minimum wages, employment protection and workfare. J. Inst. Theor. Econ. 163, 30-45 (2007)

Heckman, J.J., Smith, J.: Assessing the case for social experiments. J. Econ. Perspect. 9, 85-110 (1995)

Imbens, G., Wooldridge, J.: Recent developments in the econometrics of program evaluation. J. Econ. Lit. 47, 5-86 (2008)

Jacobebbinghaus, P., Seth, S.: The German integrated employment biographies sample IEBS. Schmollers Jahrb. Wirtsch.- Soz.wiss. 127, 335-342 (2007)

Lee, M.-J.: Micro-Econometrics for Policy, Program and Treatment Effects. Oxford University Press, Oxford (2005)

Moffitt, R.A.: The role of randomized field trials in social science research: a perspective from evaluation of reforms of social welfare programs. Am. Behav. Sci. 47, 506-540 (2004)

Ochel, W.: Welfare to work in the U.S.: A model for Germany? Finanzarchiv 59, 91-119 (2003)

Schneider, H., Lang, C., Rosenfeld, M.T.W., Kempe, W., Kolb, J.: Anreizwirkungen der Sozialhilfe auf das Arbeitsangebot im Niedriglohnbereich. Nomos Verlagsgesellschaft, Baden-Baden (2002)

Schneider, H., Uhlendorff, A.: Transitions from welfare to employment: does the ratio between labor income and social assistance matter? Schmollers Jahrb. Wirtsch.- Soz.wiss. 125, 51-61 (2005)

Schneider, H., Uhlendorff, A., Zimmermann, K.F.: Ökonometrie vs. Projektdesign: Lehren aus der Evaluation eines Modellprojekts zur Umsetzung des Workfare-Konzepts. Mimeo (2010)

Hilmar Schneider studierte Sozialwissenschaften und Volkswirtschaftslehre an der Universität Frankfurt/Main. Im Anschluss an sein sozialwissenschaftliches Diplom arbeitete er von 1983 bis 1987 als wissenschaftlicher Mitarbeiter am Sonderforschungsbereich „Mikroanalytische Grundlagen der Gesellschaftspolitik“. Nach seiner Promotion zum Dr. rer. pol. war er von 1987 bis 1993 Hochschulassistent am Fachbereich Wirtschaftswissenschaften der Universität Frankfurt/Main. 1994 übernahm er die Leitung der Abteilung Arbeitsmarkt am Institut für Wirtschaftsforschung Halle. In diesem Rahmen verbrachte er 1998 einen mehrmonatigen Forschungsaufenthalt als Visiting Scholar am World Economy Laboratory des Massachusetts Institutes of Technology (MIT). Im Juli 2001 wechselte er als Direktor für Arbeitsmarktpolitik zum IZA. Darüber hinaus ist er seit 2002 Research Affiliate des Deutschen Instituts für Wirtschaftsforschung (DIW Berlin). 2007 habilitierte er sich an der TU Darmstadt im Fach Volkswirtschaftslehre. Von 2006 bis 2007 war er Mitglied im Rat für Sozial- und Wirtschaftsdaten und seit 2007 ist er Mitglied der Zensuskommission. Seine Forschungsschwerpunkte umfassen neben der Arbeitsmarktpolitik unter anderem Fragen der Sozialen Sicherung, der Lohnpolitik und der Demografie. E-Mail: schneider@iza.org.

Arne Uhlendorff studierte Volkswirtschaftslehre und Soziologie an der Universität zu Köln, wo er 2002 sein Diplom erwarb. Im Anschluss arbeitete er bis 2007 als wissenschaftlicher Mitarbeiter am Deutschen Institut für Wirtschaftsforschung (DIW Berlin) und promovierte an der Freien Universität Berlin. Von 2007 bis 2009 war als wissenschaftlicher Mitarbeiter am IZA tätig, wo er außerdem als stellvertretender Programmdirektor für den Forschungsbereich Evaluation arbeitsmarktpolitischer Programme fungierte. Seit 2009 arbeitet er als wissenschaftlicher Mitarbeiter an der Universität Mannheim. Zugleich ist er Research Fellow des IZA und Research Affiliate des DIW Berlin. Seine Forschungsschwerpunkte umfassen Arbeitsmarktökonomie und angewandte Mikroökonometrie mit einem besonderen Fokus auf der Evaluation aktiver Arbeitsmarktpolitik, Beschäftigungsdynamik und Arbeitssuchverhalten. E-Mail: uhlendorff@uni-mannheim.de.

Klaus F. Zimmermann studierte Volkswirtschaftslehre und Statistik an der Universität Mannheim, wo er als Diplom-Volkswirt abschloss, promovierte und habilitierte. Von 1989-1998 war er Ordinarius für Volkswirtschaftslehre, insbesondere Wirtschaftstheorie an der Universität München und Direktor des SELAPO Center for Human Resources; von 1993-1995 war er zugleich Dekan der Volkswirtschaftlichen Fakultät der Universität München. Seit 1998 ist er Professor für Wirtschaftliche Staatswissenschaften der Universität Bonn und Direktor des Instituts zur Zukunft der Arbeit (IZA Bonn). Darüber hinaus ist er seit 2001 Honorarprofessor für Volkswirtschaftslehre der Freien Universität Berlin und seit 2006 Honorarprofessor der Renmin University of China). Seine aktuellen Tätigkeiten in wissenschaftlichen Vereinigungen umfassen den Vorsitz der Arbeitsgemeinschaft deutscher wirtschaftswissenschaftlicher Forschungsinstitute e.V. (ARGE, seit 2005), die Mitgliedschaft in der Deutschen Akademie der Naturforscher Leopoldina (seit 2001), die Mitgliedschaft im World Economic Forum's Global Agenda Council on Migration (seit 2009) und der Akademia Europaea (seit 2010). Er ist außerdem Research Fellow des Centre for Economic Policy Research (CEPR) in London (seit 1990), Associate Research Fellow des Centre for European Policy Studies (CEPS) in Brüssel (seit 2001), Research Associate des Center for Comparative Immigration Studies (CCIS) der University of CaliforniaSan Diego (seit 2001) und Fellow der European Economic Association (seit 2004).

Er war außerdem CORE Research Fellow 1986 (Université Catholique de Louvain, Louvain la Neuve, Belgien), Research Fellow am Wissenschaftszentrum Berlin (WZB), 1986, Visiting Associate Professor, University of Pennsylvania, Philadelphia, USA, 1987, Heisenberg Fellow, 1988-1989 und hielt die Picard-Vorlesung 1994 am Dartmouth College, USA. Seine Stationen umfassen Gastprofessuren an den Universitäten Dortmund und München, 1989, der Humboldt Universität zu Berlin, 1991, der Kyoto Universität, Japan, 1995, dem Dartmouth College, USA, 1997 und erneut der Universität München 1998. 1998 wurde ihm der Distinguished John G. Diefenbaker Award des Canada Council for the Arts verliehen.

Zimmermann ist Begründer der European Society for Population Economics (ESPE), war 1994 Präsident der European Society for Population Economics, sowie von 1991-1998 Programmdirektor für „Human Resources“ und von 1998-2001 Programmdirektor für „Labour Economics“ des CEPR (Centre for Economic Policy Research) in 
London. Lange Jahre war er Mitglied des Vorstandes der European Economic Association, der European Society for Population Economics, des Vereins für Socialpolitik und Vorsitzender des Beirates des Sozio-oekonomischen Panels (SOEP) des Deutschen Instituts für Wirtschaftsforschung (DIW Berlin), Berater des Präsidenten der EUKommission von 2001-2003 und von 2005-2009, des Ministerpräsidenten von Nordrhein-Westfalen (2008-2010) sowie zahlreicher nationaler Regierungen und Abteilungen der EU-Kommission. Von 20002011 leitete er als Präsident das Deutsche Institut für Wirtschaftsforschung.

Auch als Herausgeber von Zeitschriften hat er sich einen Namen gemacht: So ist er seit 1988 Editor-in-Chief des Journal of Population Economics, war von 1995-1998 Managing Editor von Economic Po- licy und ist bzw. war Mitherausgeber der folgenden Zeitschriften: Recherches Economiques de Louvain (seit 1991), Journal of Applied Econometrics (1992-2003), Labour Economics (1992-2000), European Economic Review (1993-1998), International Journal of Manpower (seit 1998), Economic Bulletin (2000-2007), DIW-Wochenbericht (2000-2011), DIW-Vierteljahrshefte (2002-2011), Wirtschaftsdienst (seit 2007), Applied Economics Quarterly (2008-2011) und International Encyclopedia of the Social and Behavioral Sciences (Section Editor, Labor Studies, 2nd Edition; seit 2011).

Seine Forschungsinteressen liegen im Bereich Arbeitsökonomie, Bevölkerungsökonomie, Migration, Industrieökonomie und Ökonometrie. 